\title{
Some problems in the counting of lattice animals, polyominoes, polygons and walks
}

\author{
ANDREW DANIEL REChNITZER
}

The enumeration of polyominoes and lattice site and bond animals is a famous problem in enumerative combinatorics. General polyominoes and animals of arbitrary size have not been enumerated on any regular lattice in two or more dimensions; in fact after more than 40 years of intensive study there are still very few rigorous results. In light of the difficulty of these problems it is not unreasonable to ask what we may do in order to make some progress towards a solution. Three possible options we explore in this thesis are:

1. analyse the problem numerically,

2. determine properties of the solution without actually obtaining the solution, and

3. solve similar problems.

Roughly speaking this thesis is divided into five problems falling across these three areas.

We develop a method for the investigation of certain properties of anisotropic generating functions of families of bond animals on the square lattice, including many important unsolved models. It has been suggested (on the basis of numerical evidence) that some of these properties are intimately linked with the solvability of a model. In the case of self-avoiding polygons we are able to use this method and refine its results to prove certain facts about the analytic structure of the generating function. These imply that the anisotropic generating function of self-avoiding polygons is not differentiably-finite.

The most elegant technique (to date) for the enumeration of directed animals is based on a correspondence between these animals and certain heaps of dimers, called pyramids. By extending this correspondence we are able to define and solve several new classes of polyominoes on the square and triangular lattices that are exponentially more numerous than any previously solved.

The study of polyominoes has mainly focused on their enumeration according to their most basic geometric properties - area and perimeter - and a large number

Received 12th November, 2002

Thesis submitted to the University of Melbourne, August 2000. Degree approved September 2001. Supervisors: Professor A.J. Guttman, Dr A.L. Owczarek and Dr M. Bousquet-Mélou.

Copyright Clearance Centre, Inc. Serial-fee code: 0004-9727/03 \$A2.00+0.00. 
of models have been enumerated according to both of these parameters. By contrast there are very few results concerning the site-perimeter of polyominoes; site-perimeter is of considerable interest to physicists since it plays an important role in the study of percolation models. Using a variation of the column-by-column construction known as the "Temperley method", we solve bargraph polygons according to their site-perimeter; all previous site-perimeter results concern families of convex polygons.

We investigate functional symmetries, known as "reciprocity" and "inversion relations", in the generating functions of a number of families of polygons, including several families of column-convex polygons, three-choice polygons and staircase polygons with a staircase hole. In so doing, we establish a connection between the reciprocity results known to combinatorialists and the inversion relations used by physicists to solve models in statistical mechanics. For several classes of convex polygons, the inversion (reciprocity) relation, in conjunction with certain symmetry and analyticity properties, completely determines the anisotropic perimeter generating function.

We investigate restricted self-avoiding walk models in three dimensions; in particular we examine the effect that small changes to the model have on its asymptotic behaviour. The two-dimensional case has been well studied and previously it has been argued that the universality class (that is, the dominant asymptotic behaviour) of the restricted walk model is determined primarily by the symmetries of the restricting rule. In order to search for a connection between the symmetries of the rules and possible new universality classes in three-dimensions, we have chosen a number of different restricted self-avoiding walk models and analysed them using computer enumeration and series analysis. We argue that no such link exists in three dimensions and we conjecture that there exists only one important universality class.

Department of Mathematics and Statistics

The University of Melbourne

Parkville Vic 3052

Australia 